\title{
Memantine Attenuates Salicylate-induced Tinnitus Possibly by Reducing NR2B Expression in Auditory Cortex of Rat
}

\author{
Chul Ho Jang ${ }^{1}$, Sueun Lee ${ }^{2}$, Il Yong Park ${ }^{3}$, Anji Song ${ }^{4,5}$, \\ Changjong Moon ${ }^{2 *}$ and Goang-Won Cho ${ }^{4,5 *}$ \\ ${ }^{1}$ Department of Otolaryngology, Chonnam National University Medical School, Gwangju 61469, ${ }^{2}$ Department of Veterinary \\ Anatomy, College of Veterinary Medicine and Animal Medical Institute, Chonnam National University, Gwangju 61186, \\ ${ }^{3}$ Department of Biomedical Engineering, Dankook University College of Medicine, Cheonan 31116, ${ }^{4}$ Department of Biology, \\ College of Natural Science, Chosun University, Gwangju 61452, ${ }^{5}$ Department of Life Science, BK21-Plus Research Team for \\ Bioactive Control Technology, Chosun University, Gwangju 61452, Korea
}

\begin{abstract}
Memantine, a noncompetitive antagonist of the $N$-methyl-D-aspartate (NMDA) receptor, suppresses the release of excessive levels of glutamate that may induce neuronal excitation. Here we investigated the effects of memantine on salicylate-induced tinnitus model. The expressions of the activity-regulated cytoskeleton-associated protein $(A R C)$ and tumor necrosis factor-alpha (TNFa) genes; as well as the NMDA receptor subunit $2 B$ (NR2B) gene and protein, were examined in the SH-SY5Y cells and the animal model. We also used gap-prepulse inhibition of the acoustic startle reflex (GPIAS) and noise burst prepulse inhibition of acoustic startle, and the auditory brainstem level (electrophysiological recordings of auditory brainstem responses, ABR) and NR2B expression level in the auditory cortex to evaluate whether memantine could reduce salicylate-mediated behavioral disturbances. $N R 2 B$ was significantly upregulated in salicylate-treated cells, but downregulated after memantine treatment. Similarly, expression of the inflammatory cytokine genes $T N F \alpha$ and immediate-early gene $A R C$ was significantly increased in the salicylate-treated cells, and decreased when the cells were treated with memantine. These results were confirmed by NR2B immunocytochemistry. GPIAS was attenuated to a significantly lesser extent in rats treated with a combination of salicylate and memantine than in those treated with salicylate only. The mean ABR threshold in both groups was not significant different before and 1 day after the end of treatment. Additionally, NR2B protein expression in the auditory cortex was markedly increased in the salicylate-treated group, whereas it was reduced in the memantine-treated group. These results indicate that memantine is useful for the treatment of salicylate-induced tinnitus.
\end{abstract}

Key words: Tinnitus, Salicylate, Memantine, Salicylate-induced tinnitus

Received May 21,2019, Revised August 3,2019,

Accepted August 5, 2019

\footnotetext{
* To whom correspondence should be addressed. Changjong Moon, TEL: 82-62-530-2838, FAX: 82-62-530-2809

e-mail:moonc@chonnam.ac.kr

Goang-Won Cho, TEL: 82-62-230-6641, FAX: 82-62-230-6650

e-mail:gwcho@chosun.ac.kr
}

\section{INTRODUCTION}

Tinnitus is defined as a subjective perception of sound, even though there is no actual corresponding sound stimulus. The cause of tinnitus could be synaptic excitation between the inner hair cells and the auditory nerve, although it seems to be due to a
Copyright $\odot$ Experimental Neurobiology 2019. www.enjournal.org
This is an Open Access article distributed under the terms of the Creative Commons Attribution Non-Commercial License (http://creativecommons.org/licenses/by-nc/4.0) which permits unrestricted non-commercial use, distribution, and reproduction in any medium, provided the original work is properly cited. 
reduction in the inhibitory effect on synaptic excitation. Neuronal hyperactivity of the auditory brain region is also presumed to be one of the etiological factors [1]. The salicylate-induced tinnitus model has been used to identify the biological mechanism of tinnitus ever since its introduction as a behavioral model [26]. Although the exact mechanism of tinnitus remains unknown, studies have suggested that the glutamate excitotoxicity triggered by the excitation of $N$-methyl-D-aspartate (NMDA) receptor (NR) activity via NR stimulation may be a potential risk factor for the condition [6]. Although high doses of salicylate increase the threshold of the compound action potential, salicylate paradoxically results in hyperactivity in the central auditory cortex [7]. $N M D A$ receptor subunit $2 B$ (NR2B), one of the NR subunits, may play a crucial role in tinnitus $[8,9]$.

Memantine, a noncompetitive antagonist of NR, suppresses the release of excessive levels of glutamate that may induce neuronal excitation [10]. The published studies of the effect of memantine on tinnitus are few and the results are contradictory [11-14]. Moreover, reported studies have only focused on the behavioral manifestations related to memantine.

Glutamate is known to be the chief excitatory neurotransmitter in the central nervous system and in the enteric nervous system [15]. Recently, Hwang et al. [10] reported that the mRNA expression levels of the NR2B and cytokine genes were significantly elevated in a salicylate-induced tinnitus model.

To date, it is known that immediate-early gene expression, especially that of activity-regulated cytoskeleton-associated protein (ARC/ARG3.1), c-Fos, and the known neuronal activity marker early growth response 1 (EGR-1), appears to be highly correlated with sensory-evoked neuronal activity [16]. However, the correlation between immediate-early gene expression and salicylateinduced tinnitus has been poorly studied. Recently, as reviewed by Hu et al [11], Hwang et al [10] reported the altered expression of the Arc, Egr-1, and tumor necrosis factor-alpha (TNF- $\alpha$ ) genes in the central auditory pathway in an animal model of salicylateinduced tinnitus. For a better understanding of the therapeutic effect of memantine on salicylate-induced tinnitus, observations at the cellular and auditory cortex levels would be ideal. The purpose of this study was to investigate the effects of memantine on the expression of the $A R C, E G R-1, c-F o s$, and $T N F-\alpha$ genes, as well as of the NR2B gene and protein, in the SH-SY5Y cell line. For the in vivo study, we used gap-prepulse inhibition of the acoustic startle reflex (GPIAS) and noise burst prepulse inhibition of acoustic startle (NBPIAS), as well as measurements of the auditory brainstem level (electrophysiological recordings of auditory brainstem responses, $\mathrm{ABR}$ ) and $\mathrm{NR} 2 \mathrm{~B}$ expression in the auditory cortex, to evaluate whether memantine could reduce salicylate-mediated behavioral disturbances.

\section{MATERIALS AND METHODS}

\section{Cell culture}

SH-SY5Y human neuroblastoma cells were cultured in a 100$\mathrm{mm}$ dish (SPL Life Sciences, Pocheon, Gyeonggi-do, South Korea) according to the manufacturer's recommendations. The growth medium contained 10\% fetal bovine serum (FBS) (Thermo Fisher Scientific, Mississauga, ON, Canada) and 1\% Pen Strep (Gibco, Grand Island, NY, USA) in Dulbecco's modified Eagle’s medium (Gibco, USA). For neuronal differentiation, the growth medium was replaced with differentiation medium, which contained $0.1 \%$ FBS, $1 \%$ Pen Strep, and $1 \mu \mathrm{M}$ retinoic acid (Sigma, St. Louis, MO, USA), and the cells were then further incubated for 2 days.

\section{RT-PCR and real-time PCR}

The total RNA was extracted from the cells treated as indicated, using RNAiso Plus (TAKARA, Tokyo, Japan). cDNAs were prepared using PrimeScript II $1^{\text {st }}$ Strand cDNA Synthesis kits (TAKARA, Japan) with the supplied buffer $(0.2 \mu \mathrm{g}$ of random primers, $1 \mathrm{mM}$ dNTPs). PCRs were performed with human gene-specific primers for $N R 2 B, T N F \alpha, A R C$, and $\beta$-actin (using the Power SYBR Green PCR Master Mix; Applied Biosystems, Carlsbad, CA, USA), which were synthesized by Genotech (Daejeon, South Korea) and Integrated DNA Technologies Inc. (Coralville, IA, USA). The primer information is summarized in Table 1.

Table 1. Oligonucleotides used for real-time PCR

\begin{tabular}{llll} 
Gene & \multicolumn{1}{c}{ Forward primer $\left(\mathbf{5}^{\prime} \rightarrow \mathbf{3}^{\prime}\right)$} & \multicolumn{1}{c}{ Reverse primer $\left(\mathbf{5}^{\prime} \rightarrow \mathbf{3}^{\prime}\right)$} & Acc. $\mathbf{~ N o .}$ \\
\hline$A R C$ & ACAACAGGTCTCAAGGTTCCC & AGCCGACTCCTCTCTGTAGC & NM_015193.4 \\
$N R 2 B$ & GGAGAGGTGGTCATGAAGAG & CATTGCTGCGTGACACCATG & NM_000834.4 \\
$T N F \alpha$ & TGGGAGCTTGATTCTCAGCA & CCTGGGCTTGACCTCTCTGTA & NM_000594.3 \\
$\beta$-actin & ATCCGCAAAGACCTGTACGC & TCTTCATTGTGCTGGGTGCC & NM_001101 \\
\hline
\end{tabular}

Acc. No. indicates the gene accession number. 


\section{Immunocytochemical staining}

SH-SY5Y cells were grown on coated coverslips (Thermo Fisher Scientific, Waltham, MA, USA) and differentiated into neuron-like cells. The cells were treated with $0.25 \mu \mathrm{g} / \mathrm{mL}$ memantine for $6 \mathrm{~h}$ and then treated with $40 \mu \mathrm{g} / \mathrm{mL}$ aspirin for $8 \mathrm{~h}$. Thereafter, the cells were stained with primary antibody against NR2B (1:900; Invitrogen, Carlsbad, CA, USA) for $2 \mathrm{~h}$ at room temperature (RT), and then with the donkey anti-rabbit IgG secondary antibody (1:400, conjugated with Alexa 555; Molecular Probes Inc., Eugene, OR, USA) together with Hoechst 33342 (1:1000; Molecular Probes Inc., USA) for $1 \mathrm{~h} 30 \mathrm{~min}$ at RT. After washing with phosphatebuffered saline (PBS), the cells were mounted (ProLong Gold antifade reagent; Molecular Probes Inc., USA) and visualized under a Nikon Eclipse Ti2 fluorescence microscope (Nikon, Tokyo, Japan). Cell images were taken with a DS-Ri2 digital camera (Nikon, Japan).

\section{In vivo study}

\section{Animals}

Experiments were designed using 20 adult male Sprague-Dawley rats (weighing $300 \mathrm{~g}$ ) with normal eardrums. All procedures were approved by the Institutional Animal Care and Use Committee (CNU IACUC-H-2018-55). The animals were randomly divided into either a control group $(n=10)$ or a study group $(n=10)$ for GPIAS, NBPIAS, and ABR evaluations. The control group was used to evaluate salicylate-induced tinnitus, and the study group to assess the therapeutic effect of memantine on salicylate-in- duced tinnitus. The control group was injected intraperitoneally (IP) with $400 \mathrm{mg} \cdot \mathrm{kg}^{-1} \cdot \mathrm{day}^{-1}$ of sodium salicylate, whereas the study group was injected IP with sodium salicylate combined with 5 $\mathrm{mg} \cdot \mathrm{kg}^{-1} \cdot \mathrm{day}^{-1}$ of memantine (Sigma, USA) for 7 consecutive days.

\section{Gap and noise burst prepulse inhibition of acoustic startle}

A startle response measurement system was used to obtain the GPIAS and NBPIAS values. As shown in Fig. 1A, the system was composed of a mesh cage with a vibration sensor, a noise box with an anechoic inner wall, an acoustic stimulator (PM-5004 amplifier; Marantz, Kawasaki, Japan, with a full-range loud speaker), a reference microphone (40PH; GRAS, Holte, Denmark), sensor signal acquisition hardware (PC and NI PCIe-6321; National Instruments, Austin, TX, USA), and LabVIEW-based custom graphical user interface (GUI) software. An accelerometer sensor module (LIS344ALH; STMicroelectronics, Geneva, Switzerland) for sensing the startling vibration in rats was attached at the bottom plate of the mesh cage. Our implemented GUI software controlled all of the processes for acquiring startle responses through acoustic stimulation, and performed the analysis for obtaining GPIAS and NBPIAS values. All the measurements for the startle responses were obtained using the integrated system in the soundproof box (Fig. 1A). For the GPIAS measurement, a session with each rat involved 15 startle responses evoked by gap-conditioned stimuli and another 15 startle responses evoked by a stimulus with no gap. In addition, two kinds of acoustic stimuli (a gap stimulus and a nogap stimulus) were presented to rats in randomly ordered pairs. The intervals of the acoustic stimuli were changed randomly from
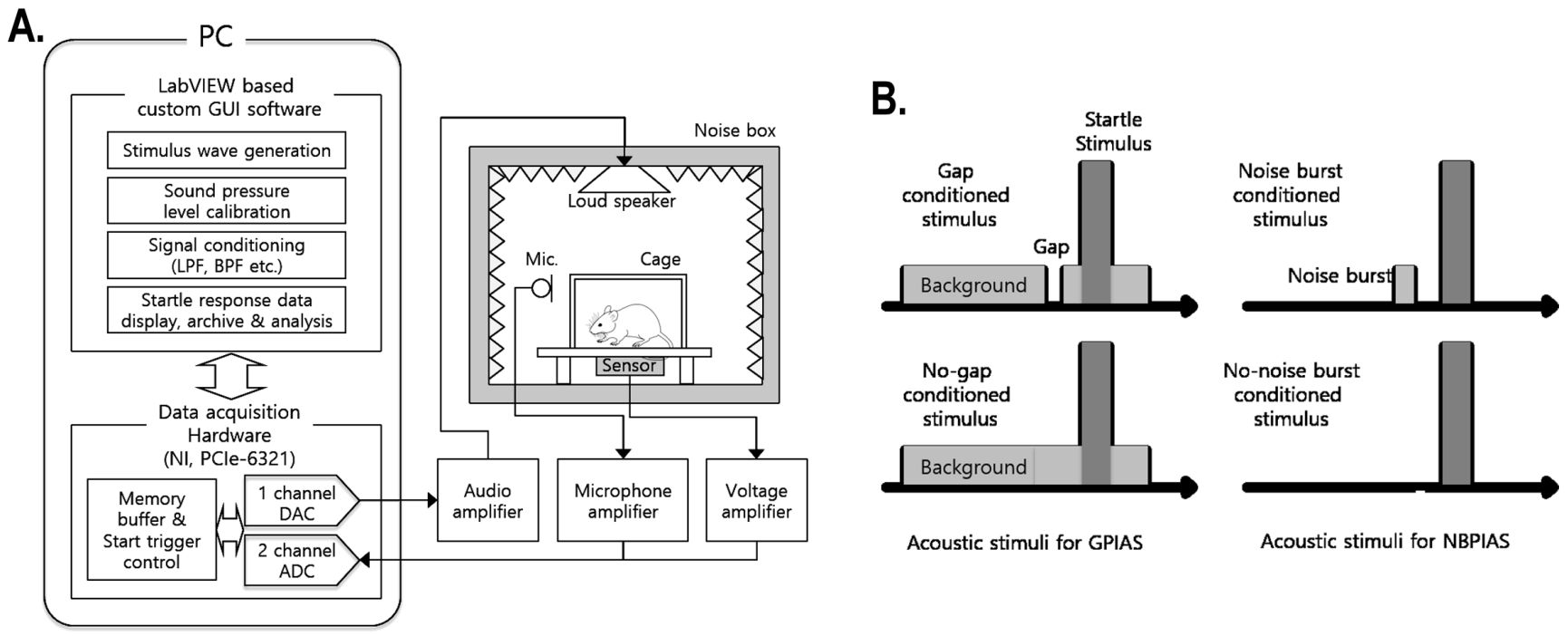

Fig. 1. Schematic diagram of the experimental instrument and schedule for investigation. (A) NI, National Instrument; DAC, digital-to-analog converter; ADC, analog-to-digital converter; Mic, microphone. (B) GPIAS, gap prepulse inhibition of acoustic startle reflex; NBPIAS, noise burst prepulse inhibition of acoustic starte reflex. 
$17 \mathrm{~s}$ to $23 \mathrm{~s}$. Before beginning the first session, the rat was placed in the cage for $2 \mathrm{~min}$ to acclimatize it to the measurement environment. The acoustic stimulation for the GPIAS consisted of narrowband background noise ( $1 \mathrm{kHz}$ bandwidth, $16 \mathrm{kHz}$ center frequency, and $60 \mathrm{~dB}$ sound pressure level [SPL]), with a short highlevel stimulus for startling (broadband noise burst, 105 dB SPL, 50 ms length) and a prepulse gap inserted before the startle stimulus (50 ms length, onset on $100 \mathrm{~ms}$ prior to the onset of startle stimulus), as shown in Fig. 1B.

After GPIAS measurement, NBPIAS test was also measured. The main object of NBPIAS is to assess the animal hearing function. Because the hearing loss is the potential factor which affects the result of GPIAS measurement. If the hearing loss caused by salicylate treatment, it can make the background sound and the embedded gaps less audible. Moreover, hearing loss can also attenuate startle reflex magnitude, therefore GPIAS cannot be used for tinnitus assessment. NBPIAS recording was performed same as GPIAS measurement equipment.

For NBPIAS, a narrowband noise burst prepulse $(50 \mathrm{~ms}$ length, $1 \mathrm{kHz}$ bandwidth, $16 \mathrm{kHz}$ center frequency, and $60 \mathrm{~dB}$ SPL) was used instead of a prepulse gap and without background noise (Fig. 1B). In addition, 15 startle responses to the prepulse condition stimulus and another 15 responses to the no-prepulse stimulus were obtained with the same recording configuration as used for GPIAS. The outlying startle responses measured in each stimulation condition group were eliminated using Grubbs' test. Root mean square (RMS) value is still the effective for noise or signal bursts. It is commonly used to compute a single value that represents the composite of different sine wave components. GPIAS and NBPIAS were defined as the inhibition percentages, calculated as (1-RMS of gap startle response/RMS of no gap startle response $) \times 100$ and ( $1-$ RMS of noise burst response/RMS of no noise burst response) $\times 100$, respectively. A high GPIAS or NBPIAS percentage value meant that a gap or a noise burst prepulse had inhibited the startle response. A rat with tinnitus at approximately $16 \mathrm{kHz}$ typically shows a significantly lower GPIAS value because the animal cannot distinguish between the gap prepulse and the background sound owing to the tinnitus. A low NBPIAS value indicates that the rat could not hear the noise burst prepulse of the same SPL and frequency as the background noise used in the GPIAS measurement. Therefore, a rat with a low GPIAS and a normal NBPIAS value can be considered as being a model of induced tinnitus without hearing loss [17]. All the animals were tested for GPIAS and NBPIAS before sodium salicylate injection and $2 \mathrm{~h}$ afterwards. GPIAS measurements were performed continuously on a daily basis from 0 to day 8 and NBPIAS measurements were taken at day $0,1,3,5,7$, and 8 (Fig. 2).

\section{Measurement of auditory brainstem responses}

We evaluated hearing function using an ABR measuring system (Tucker-Davis Technologies, Miami, FL, USA). ABR thresholds were obtained in all animals before the first drug treatment and on day 8 following it. The procedure used for recording the ABR was the same as described in our previous publication [18]. In brief, after placement of the subdermal needle electrodes at the vertex and the reference electrode at the occiput, the hearing thresholds were assessed using tone bursts ( $2 \mathrm{~ms}$ rise/decay, $1 \mathrm{~ms}$ plateau), presented at a rate of 50/s in a decreasing intensity series, beginning with levels that elicited distinct evoked potentials. The stimulus was started at $90 \mathrm{~dB}$ SPL and repeated at intervals of $10 \mathrm{~dB}$ in a downward progression until no response was identifiable.

\section{Preparation of free-floating sections}

Rats were sacrificed 7 days after the injection of each drug or vehicle for histological examination of the brain tissue. The animals were anesthetized and perfused with $4 \%(\mathrm{w} / \mathrm{v})$ paraformaldehyde (PFA) in PBS (pH 7.4), following which the brains were removed immediately and stored in 4\% (w/v) PFA in PBS for 2 days at $4{ }^{\circ} \mathrm{C}$. The brains were suspended in $30 \%(\mathrm{w} / \mathrm{v})$ sucrose for 4 days and then embedded in optimum cutting temperature compound (Miles Inc., Elkhart, IN, USA). Using a sliding microtome (SM2010R; Leica Microsystems, Wetzlar, Germany), the hemispheres were coronally sectioned at approximately $4.30 \sim 5.30 \mathrm{~mm}$ caudal to the bregma for the auditory cortex. Free-floating serial sections $(30 \mu \mathrm{m}$ thick) were collected into 10 wells filled with PBS.

\section{Immunohistochemistry of free-floating sections}

The free-floating sections were incubated with $0.3 \%(\mathrm{v} / \mathrm{v})$ hydrogen peroxide in distilled water for $20 \mathrm{~min}$, and then blocked with

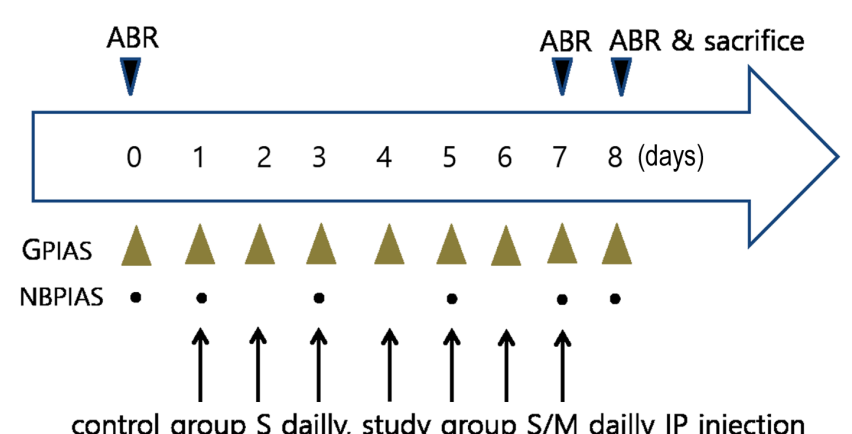

control group S dailly, study group S/M dailly IP injection

Fig. 2. Schedule for investigation of the effect of memantine. Drug was administered from day 1 to day 7 (S: salicylate, M: memantine). GPIAS (gap prepulse inhibition of acoustic startle reflex) and NBPIAS (noise burst prepulse inhibition of acoustic startle reflex) performed from day 0 , to day 8. ABR (auditory brainstem response) was performed on day 0 , day 7 and 8 . 
$5 \%(\mathrm{v} / \mathrm{v})$ normal goat serum in PBS with $0.3 \%(\mathrm{v} / \mathrm{v})$ Triton X-100 (PBS-T) for $1 \mathrm{~h}$ at RT. The sections were incubated with rabbit anti-NR2B (1:500 dilution) primary antibody in antibody dilution buffer (Invitrogen, USA) for 1 day at $4{ }^{\circ} \mathrm{C}$. After washing with PBS$\mathrm{T}$, the sections were reacted with biotinylated goat anti-rabbit IgG (Vector ABC Elite Kit; Vector Laboratories, Burlingame, CA, USA) for $1 \mathrm{~h}$ at RT, washed with PBS-T, and incubated for $1 \mathrm{~h}$ at RT with an avidin-biotin peroxidase complex (Vector ABC Elite Kit). After washing with PBS-T, the peroxidase reaction was performed using the diaminobenzidine substrate (contained in the DAB kit; Vector Laboratories). Immunohistochemically stained specimens were examined using a microscope (Olympus, Tokyo, Japan) fitted with an eXcope X3 digital camera (DIXI Optics, Daejeon, South Korea).

\section{Statistical analysis}

Analysis of variance followed by Bonferroni post hoc test was performed. The data represent the mean \pm SEM.

\section{RESULTS}

\section{In vitro study}

To examine the effect of memantine on salicylate-treated neuronal cells, SH-SY5Y cells were differentiated into neuron-like cells and treated with $0.25 \mu \mathrm{g} / \mathrm{mL}$ memantine for $6 \mathrm{~h}$ followed by treatment with $40 \mu \mathrm{g} / \mathrm{mL}$ salicylate for $8 \mathrm{~h}$, and the $N R 2 B$ expression level was then measured by real-time PCR. $N R 2 B$ expression was significantly increased in salicylate-treated cells but decreased after memantine treatment (Fig. 3). Similarly, expression of the inflammatory cytokine $T N F \alpha$ and immediate-early $A R C$ genes was increased in salicylate-treated cells, and decreased when the cells were treated with memantine (Fig. 3). We confirmed these results through immunocytochemical staining with the NR2B antibody and obtained consistent results (Fig. 4).

\section{In vivo study}

\section{GPIAS and NBPIAS}

During the baseline sessions, the mean GPIAS value was $46.3 \%$ in the control group and $47.2 \%$ in the study group. As shown in

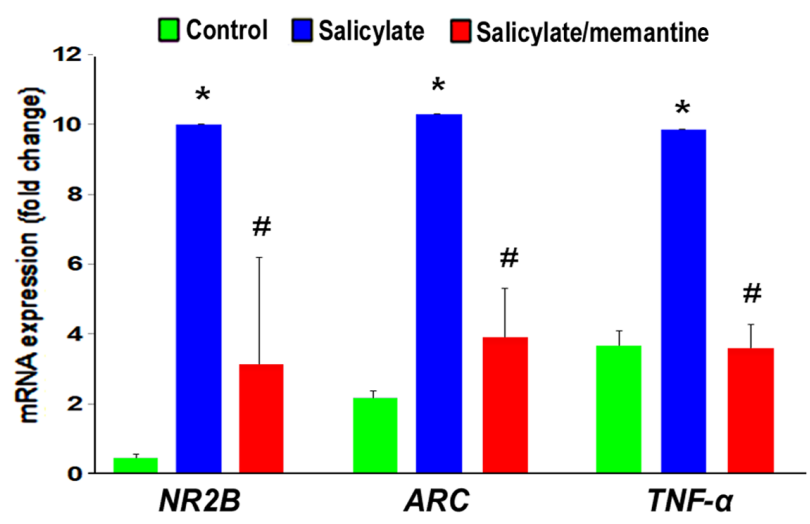

Fig. 3. The gene expressions in the salicylate treated cell with/without memantine treatment. The expression of NR2B $\left({ }^{*} \mathrm{p}<0.001,{ }^{*} \mathrm{p}<0.05\right.$, mean $\pm \mathrm{SD}, n=3), \operatorname{Arc}\left({ }^{*} \mathrm{p}<0.001,{ }^{*} \mathrm{p}<0.05\right.$, mean $\left.\pm \mathrm{SD}, n=6\right)$ and $T N F-\alpha$ mRNA $\left({ }^{*} \mathrm{p}<0.001,{ }^{*} \mathrm{p}<0.05\right.$, mean $\left.\pm \mathrm{SD}, n=10\right)$ was significantly lower in the memantine treatment group compared to the salicylate group. T-test, * indicates control versus salicylate. \# indicates salicylate versus salicylate/memantine.
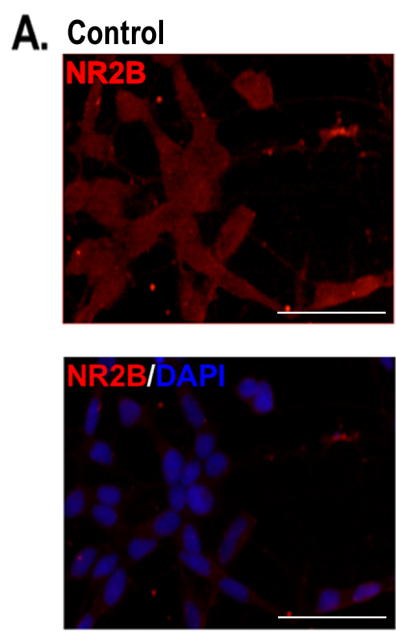

Salicylate
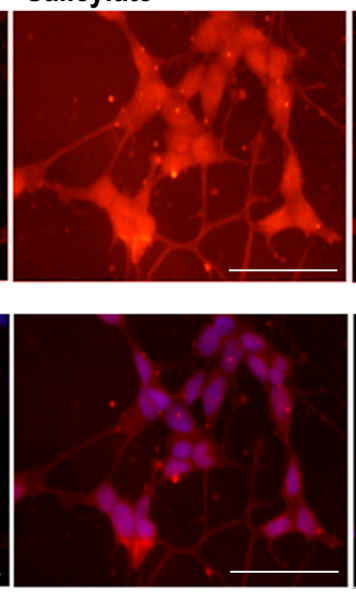

Salicylate/memantine
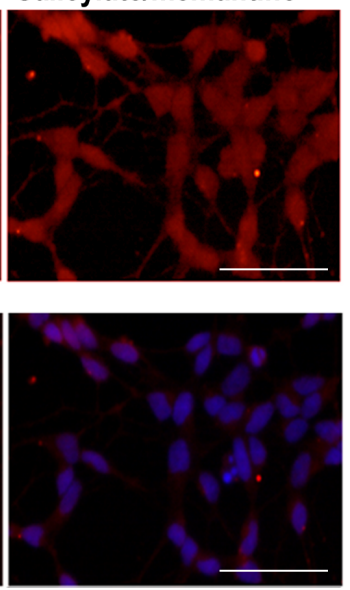

B.

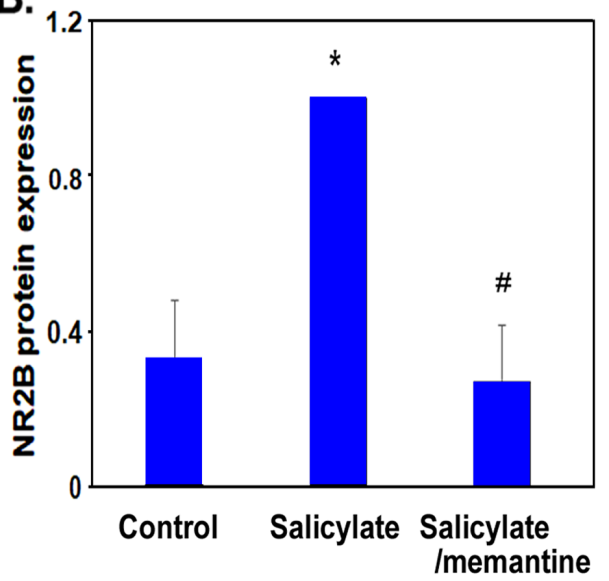

Fig. 4. Immunocytochemistry shows that the expression of NR2B was significantly lower in the memantine treatment group compared to the salicylate group. (A) Red indicates NR2B, blue indicates DAPI. Scale bar indicates $100 \mu \mathrm{m}$. (B) Protein expression levels were quantified using ImageJ software. Ttest, ${ }^{*}$ indicates control versus salicylate. \# indicates salicylate versus salicylate/memantine $\left({ }^{*} \mathrm{p}, \# \mathrm{p}<0.01\right.$, mean $\left.\pm \mathrm{SD}, n=3\right)$. 

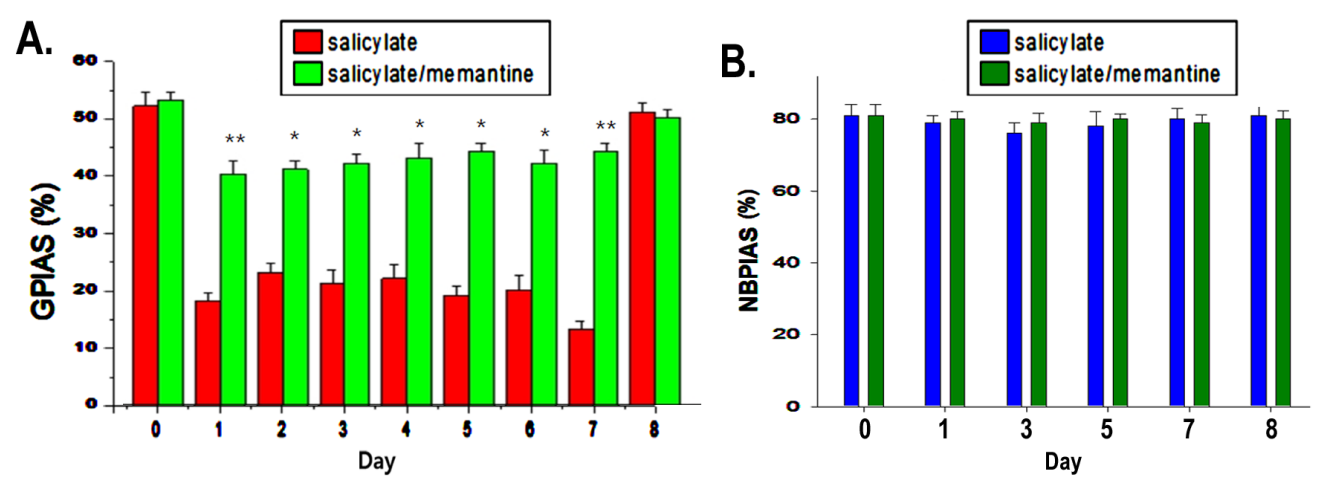

A.

click
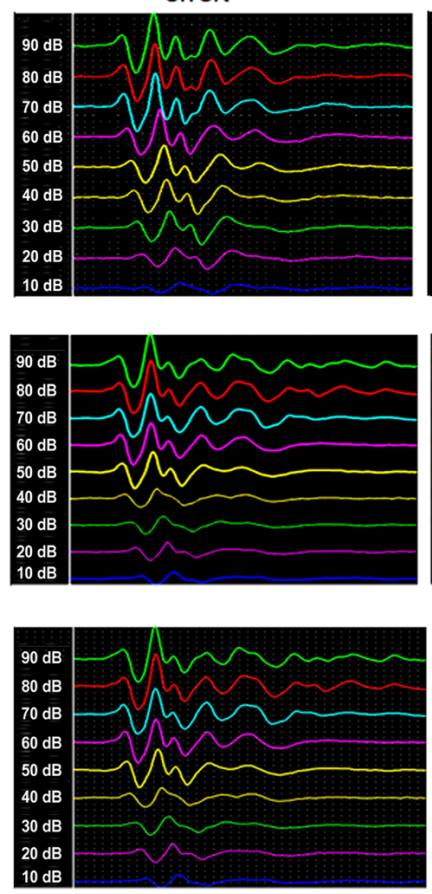

$8 \mathrm{kHz}$

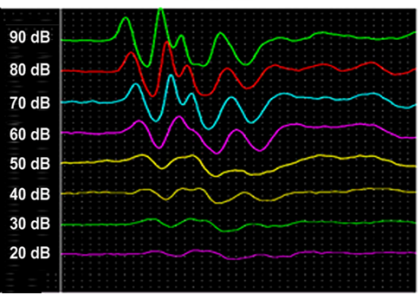

day $\mathbf{0}$, Salicylate group

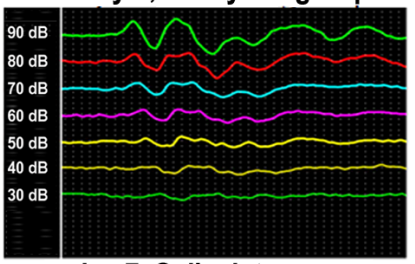

day 7 , Salicylate group

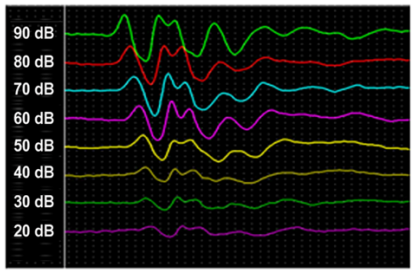

day 7 , Salicylate/memantine group

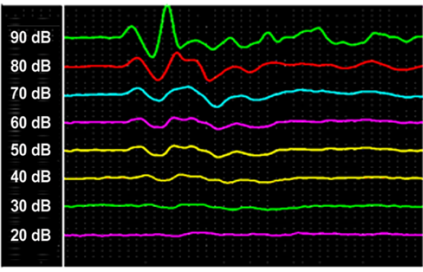

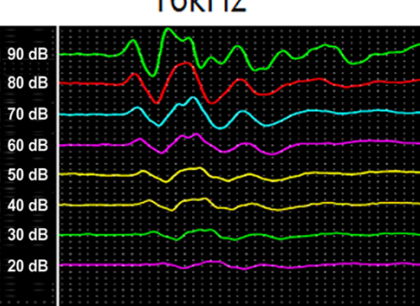

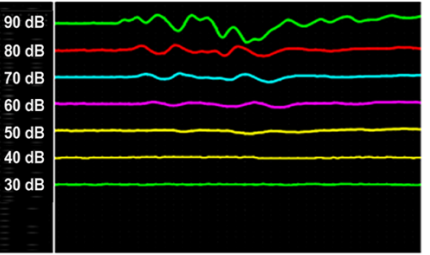

B.

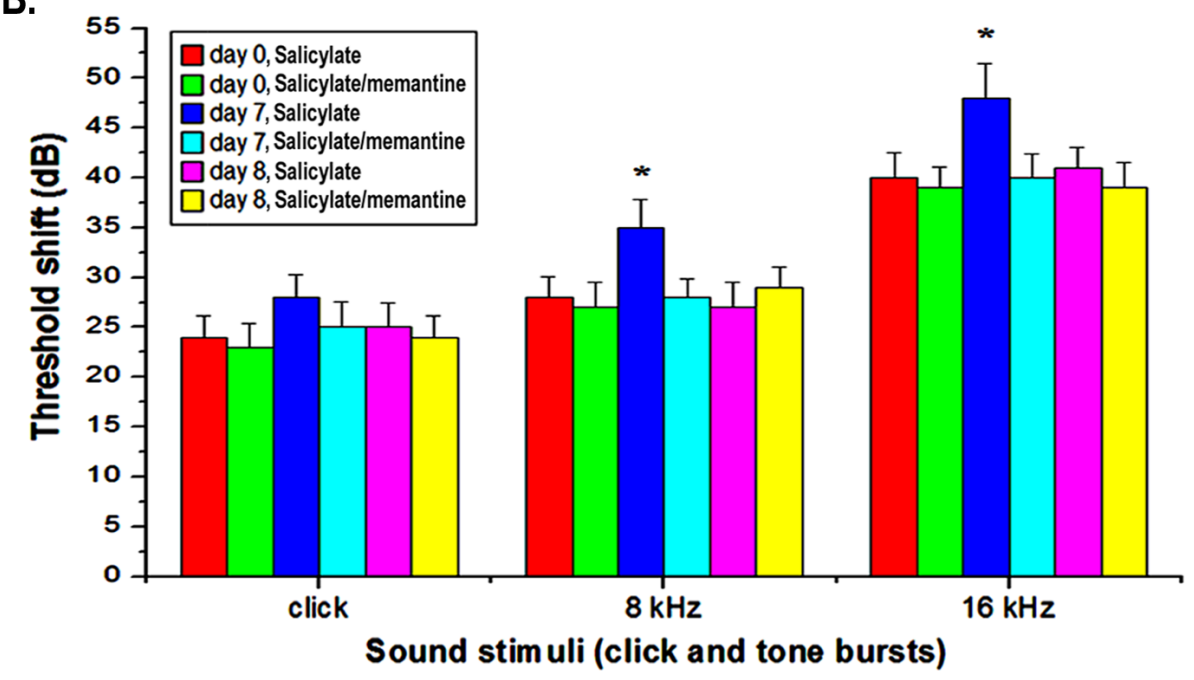

Fig. 6. The salicylate group shows that memantine attenuated the salicylate-induced threshold shift at 8 and $16 \mathrm{kHz}(\mathrm{A}$; ${ }^{*} \mathrm{p}<0.05$, mean $\left.\pm \mathrm{SD}, n=10\right)$ and the raw traces of the electrophysiological recordings (B).

GPIAS which was decreased by salicylate $\left(\mathrm{A} ;{ }^{*} \mathrm{p}<0.05,{ }^{* *} \mathrm{p}<0.01\right.$, mean $\pm S D, n=10)$. The mean NBPIAS values in both groups were not significantly different throughout the entire testing period (B; mean $\pm \mathrm{SD}, n=10)$. 
A.
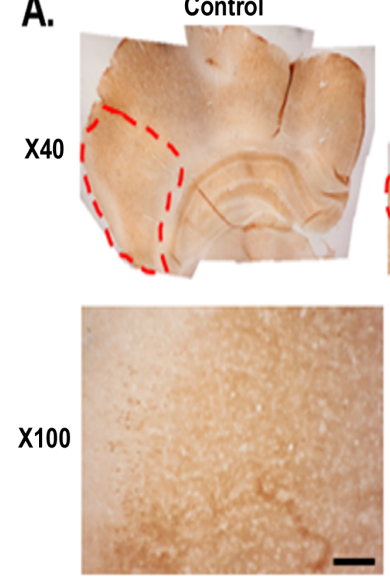
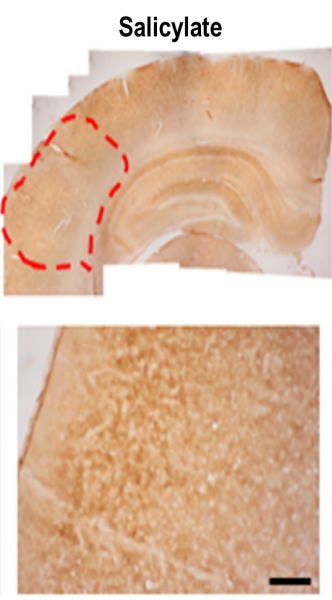
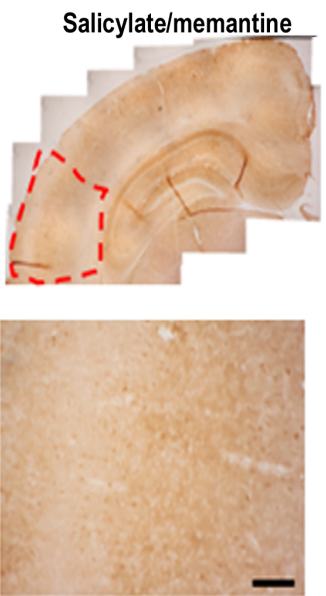

B.

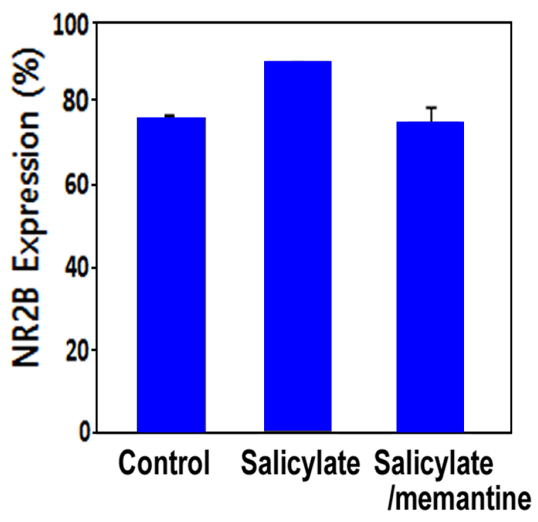

Fig. 7. NR2B-immunolabeled in the auditory cortex. (A) The dotted circle indicates the auditory cortex (mean \pm SD, $n=5$ ). (B) Protein expression levels were quantified using ImageJ software.

Fig. 5A, the mean GPIAS value was reduced in the control group, indicating that tinnitus had been induced in the rats. As compared with that in the group treated with salicylate alone, the GPIAS value attenuated to a significantly lesser extent in the group treated with a combination of salicylate and memantine $(\mathrm{p}=0.01$ at day 1 , $\mathrm{p}=0.01$ at day $3, \mathrm{p}=0.003$ at day 5 , and $\mathrm{p}=0.03$ at day 7 ). The mean NBPIAS values in both groups were not significantly different throughout the entire testing period (Fig. 5B). This was supported by the experimental results using GPIAS and audiological measurements. The GPIAS recovery rate in the study group was similar to that reported by Ralli et al [19].

\section{Auditory brainstem responses}

To determine whether salicylate could induce a temporary auditory threshold shift, ABR threshold shifts were observed before the drug treatment and on days 7 and 8 ( 1 day after the end of treatment). The salicylate group showed an elevated threshold compared with that before treatment (the asterisk indicates statistical significance, $\mathrm{p}=0.26$ ), whereas the salicylate plus memantine group showed an attenuation of the threshold shift on day 7 . The mean ABR threshold in both groups was not significant before and 1 day after the end of treatment $(\mathrm{p}=0.17)$ (Fig. 6A and 6B). These results indicated that memantine attenuated the salicylate-induced threshold shift at 8 and $16 \mathrm{kHz}$.

\section{Immunohistochemistry of the auditory cortex}

In the salicylate group, NR2B protein expression was markedly increased in the auditory cortex, whereas it was reduced in the memantine-treated group (Fig. 7).

\section{DISCUSSION}

The mRNA levels of some immediate-early genes (Arc, Egr-1, and $c$-Fos) appeared to be highly correlated with neuronal-activating stimulation in the brain, where synaptic NR activation was found to be associated with the rapid regulation of these genes [20]. Although only a few articles have been published, the correlation of immediate-early genes with the tinnitus model has been reported $[11,21,22]$. In this study, we established cell and animal models of salicylate-induced tinnitus for examination of the effects of memantine. We observed increased expression of $N R 2 B$ in the salicylate-treated cell group, and its decrease by memantine. This effect was supported by the expression levels of the inflammatory cytokine gene $T N F a$ and immediate-early gene $A R C$. The upregulation of the $T N F \alpha$ and $A R C$ genes by salicylate was similar to that in the report by Hwang et al $[10,23]$, but in the present study, the expression of the c-Fos and EGR-1 genes did not show significant differences. Hu et al [11] reported that the gene expression levels of Arc and Egr- 1 were decreased in the inferior colliculus and auditory cortex; however, in our present study, Arc gene expression was significantly upregulated in the SH-SY5Y cells.

In the present study, the GPIAS value attenuated to a significantly lesser extent in the group treated with a combination of salicylate and memantine. This was supported by the experimental results using GPIAS and audiological measurements. The GPIAS recovery rate in the study group was similar to that reported by Ralli et al [19]. Our study showed, memantine attenuated the salicylate-induced threshold shift at 8 and $16 \mathrm{kHz}$. The memantine-mediated attenuation of the salicylate-induced increase in NR2B expression in the auditory cortex may be associated with NR blocking. These 
results indicated that NR2B expression was mediated through an NMDA glutamate receptor in salicylate-induced tinnitus. The neuroprotective action of memantine through the restoration of synaptic plasticity was reported in animal models of neurodegeneration [24].

A limitation of our present study is that we did not investigate whether memantine attenuates the synaptic action between inner hair cells and auditory nerve fibers in salicylate-induced tinnitus. Recently, the beneficial effect of the intratympanic application of AM-101 (an NR antagonist) on patients with tinnitus has been reported. Further studies of memantine, especially its effects on the synaptic action between inner hair cells and the auditory nerve, are necessary.

\section{ACKNOWLEDGEMENTS}

This work was supported by a grant from the Basic Science Research Program through the National Research Foundation of Korea (NRF) funded by the Ministry of Education, Science and Technology (NRF-2018R1D1A1B07048074 and NRF-2017R1D1A1B03034527).

\section{REFERENCES}

1. Robertson D, Bester C, Vogler D, Mulders WH (2013) Spontaneous hyperactivity in the auditory midbrain: relationship to afferent input. Hear Res 295:124-129.

2. Yang K, Huang ZW, Liu ZQ, Xiao BK, Peng JH (2009) Longterm administration of salicylate enhances prestin expression in rat cochlea. Int J Audiol 48:18-23.

3. Song RB, Lou WH (2015) Monosialotetrahexosylganglioside inhibits the expression of p-CREB and NR2B in the auditory cortex in rats with salicylate-induced tinnitus. Clin Lab 61:1113-1118.

4. Wan I, Pokora O, Chiu T, Lansky P, Poon PW (2015) Altered intensity coding in the salicylate-overdose animal model of tinnitus. Biosystems 136:113-119.

5. Lauer AM, Larkin G, Jones A, May BJ (2018) Behavioral animal model of the emotional response to tinnitus and hearing loss. J Assoc Res Otolaryngol 19:67-81.

6. Guitton MJ, Caston J, Ruel J, Johnson RM, Pujol R, Puel JL (2003) Salicylate induces tinnitus through activation of cochlear NMDA receptors. J Neurosci 23:3944-3952.

7. Chen GD, Stolzberg D, Lobarinas E, Sun W, Ding D, Salvi R (2013) Salicylate-induced cochlear impairments, cortical hyperactivity and re-tuning, and tinnitus. Hear Res 295:100113.
8. Ruel J, Chabbert C, Nouvian R, Bendris R, Eybalin M, Leger CL, Bourien J, Mersel M, Puel JL (2008) Salicylate enables cochlear arachidonic-acid-sensitive NMDA receptor responses. J Neurosci 28:7313-7323.

9. Zhao J, Wang B, Wang X, Shang X (2018) Up-regulation of $\mathrm{Ca}^{2+} / \mathrm{CaMKII/CREB}$ signaling in salicylate-induced tinnitus in rats. Mol Cell Biochem 448:71-76.

10. Hwang JH, Chen JC, Yang SY, Wang MF, Liu TC, Chan YC (2011) Expression of COX-2 and NMDA receptor genes at the cochlea and midbrain in salicylate-induced tinnitus. Laryngoscope 121:361-364.

11. Hu SS, Mei L, Chen JY, Huang ZW, Wu H (2014) Expression of immediate-early genes in the inferior colliculus and auditory cortex in salicylate-induced tinnitus in rat. Eur J Histochem 58:2294.

12. Lu S, Nasrallah HA (2018) The use of memantine in neuropsychiatric disorders: An overview. Ann Clin Psychiatry 30:234-248.

13. Lobarinas E, Yang G, Sun W, Ding D, Mirza N, Dalby-Brown W, Hilczmayer E, Fitzgerald S, Zhang L, Salvi R (2006) Salicylate- and quinine-induced tinnitus and effects of memantine. Acta Otolaryngol Suppl 126:13-19.

14. Figueiredo RR, Langguth B, Mello de Oliveira P, Aparecida de Azevedo A (2008) Tinnitus treatment with memantine. Otolaryngol Head Neck Surg 138:492-496.

15. Liu MT, Rothstein JD, Gershon MD, Kirchgessner AL (1997) Glutamatergic enteric neurons. J Neurosci 17:4764-4784.

16. Flavell SW, Greenberg ME (2008) Signaling mechanisms linking neuronal activity to gene expression and plasticity of the nervous system. Annu Rev Neurosci 31:563-590.

17. Lowe AS, Walton JP (2015) Alterations in peripheral and central components of the auditory brainstem response: a neural assay of tinnitus. PLoS One 10:e0117228.

18. Jang $\mathrm{CH}$, Cho YB, Choi CH, Jang YS, Jang SJ, Jung WK, Park BY, Kim MY (2014) ALH-L1005 attenuates endotoxin induced inner ear damage. Int J Pediatr Otorhinolaryngol 78:465-470.

19. Ralli M, Troiani D, Podda MV, Paciello F, Eramo SL, de Corso E, Salvi R, Paludetti G, Fetoni AR (2014) The effect of the NMDA channel blocker memantine on salicylate-induced tinnitus in rats. Acta Otorhinolaryngol Ital 34:198-204.

20. Okuno H (2011) Regulation and function of immediateearly genes in the brain: beyond neuronal activity markers. Neurosci Res 69:175-186.

21. Tan J, Rüttiger L, Panford-Walsh R, Singer W, Schulze H, Kilian SB, Hadjab S, Zimmermann U, Köpschall I, Rohbock K, Knipper M (2007) Tinnitus behavior and hearing function 
correlate with the reciprocal expression patterns of BDNF and Arg3.1/arc in auditory neurons following acoustic trauma. Neuroscience 145:715-726.

22. Santos P, da Silva LE, Leão RM (2017) Specific immediate early gene expression induced by high doses of salicylate in the cochlear nucleus and inferior colliculus of the rat. Rev Bras Otorrinolaringol (Engl Ed) 83:155-161.
23. Hwang JH, Chen JC, Yang SY, Wang MF, Chan YC (2011) Expression of tumor necrosis factor- $\alpha$ and interleukin- $1 \beta$ genes in the cochlea and inferior colliculus in salicylate-induced tinnitus. J Neuroinflammation 8:30.

24. Greig SL (2015) Memantine ER/donepezil: a review in Alzheimer's disease. CNS Drugs 29:963-970. 